\title{
Protective properties of a missile enclosure against electromagnetic influences
}

\author{
S. Fisahn and H. Garbe \\ Leibniz Universität Hannover, Germany
}

\begin{abstract}
In order to predict the immunity of a generic missile (GENEC), not only the electronic system but also the enclosure has to be taken into consideration. While a completely closed metallic missile enclosure shows a high electric shielding effectiveness, it is decreased substantially by apertures which could not be avoided by different reasons. The shielding effectiveness of the generic missile could be investigated by means of a hollow cylinder equipped with different apertures. Numerical simulations and measurements of this hollow cylinder will be carried out and analyzed.
\end{abstract}

\section{Introduction}

Modern electronic systems are of particular importance under many aspects like security, medicine, economy, traffic, communication and armed forced. Civil and military systems consist of several electronic components and subsystems (e.g. microprocessor boards). Malfunctions of these devices could lead to unexpected consequences, since the electronic components have got safety critical functionalities. Susceptibility investigations of different electronic systems have shown that electromagnetic threats like electromagnetic pulses (EMP), ultra wideband (UWB) and high power microwave pulses (HPM) can cause failures or damages. In case of a time critical system like an aircraft, even a failure without any physical damage of an electronic component (e.g. self reset of a microcontroller) could lead to the destruction of the complete system due to a crash. In order to predict the immunity of a missile against different electromagnetic threats, not only the electronic system but also the enclosure has to be taken into consideration. While a completely closed metallic missile enclosure shows a high electric shielding ef- fectiveness, it will be decreased substantially by apertures which could not be avoided by different reasons.

In this paper, the protective properties of a missile enclosure are investigated by means of a simplified enclosure model, which is described in Sect. 2. In order to obtain the field distribution inside the missile enclosure, numerical field calculations were carried out in frequency domain. The requirements of these simulations will be presented in Sect. 3, as well as the simulation results and their evaluation. After this, several measurements of this the missile model were performed in time and frequency domain. The measurement setup and the evaluation of the measurements will be described in Sect. 4. In the following conclusion, the results of the numerical field calculations and the measurements will be compared to each other and evaluated as well.

\section{Modelling concept}

In this contribution, the investigation of the protective properties of a missile enclosure against electromagnetic influences is performed on the basis of a simplified enclosure model. Assuming that the conductivity of the enclosure walls is sufficiently, only aperture penetration affects the coupling of electromagnetic fields to the interior of the missile enclosure. Thus, the model consists of a metallic hollow cylinder equipped with an aperture at the front side. Such an opening could be necessary by different reasons e.g. for the optical component of a target tracking system. The geometrical dimensions of the hollow cylinder are $l=1 \mathrm{~m}$ and $D_{\text {cyl }}=100 \mathrm{~mm}$. Furthermore, a monopole field probe is positioned inside the hollow cylinder, in order to detect the fields inside the cylinder.

Correspondence to: S. Fisahn

(fisahn@ieee.org)

Published by Copernicus Publications on behalf of the URSI Landesausschuss in der Bundesrepublik Deutschland e.V. 


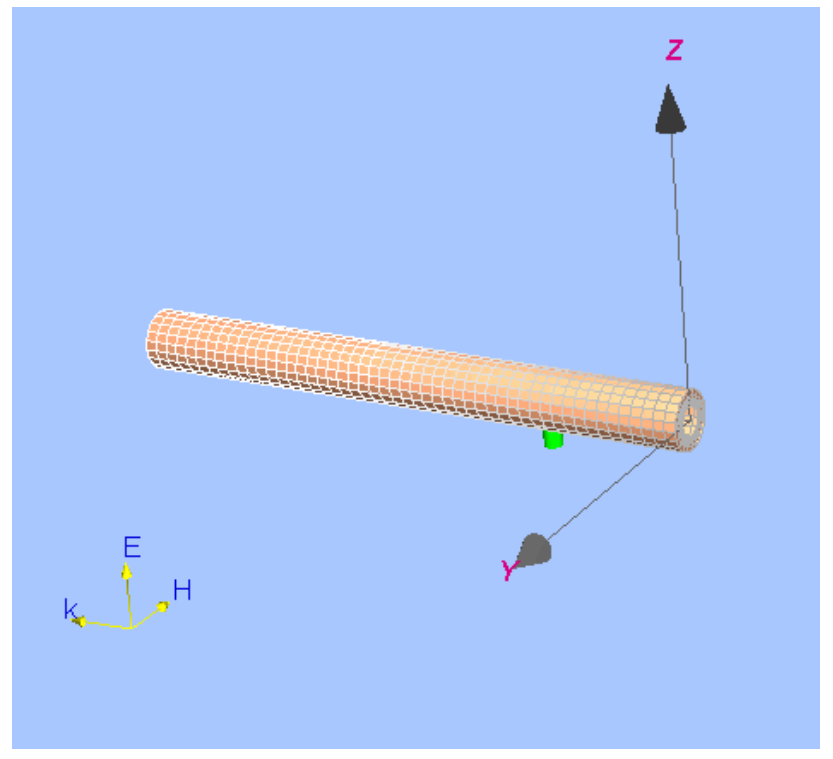

Fig. 1. Simulation model.

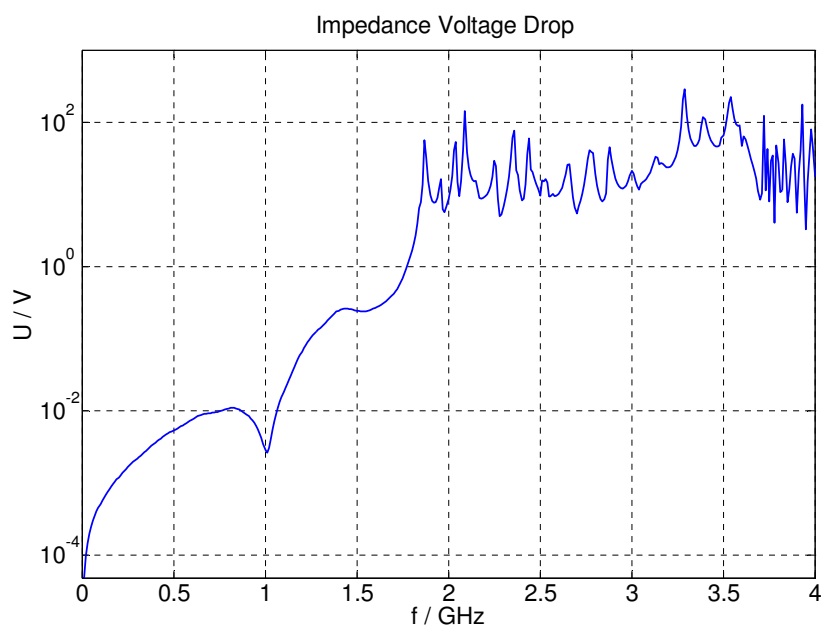

Fig. 2. Calculated impedance voltage drop at the antenna base of the monopole.

\section{Numerical field calculations}

Simulations have been carried out in frequency domain with the numerical field calculation program CONCEPT-II. The aim of these calculations is the investigation of the coupling mechanism of the electromagnetic fields from the surroundings to the interior of the missile enclosure. Additionally, the simulation results could be used in order to validate the measurement results.

\subsection{Simulation model}

The simulation model consists of an ideal conducting metallic hollow cylinder with the geometric dimensions $l=1 \mathrm{~m}$ and

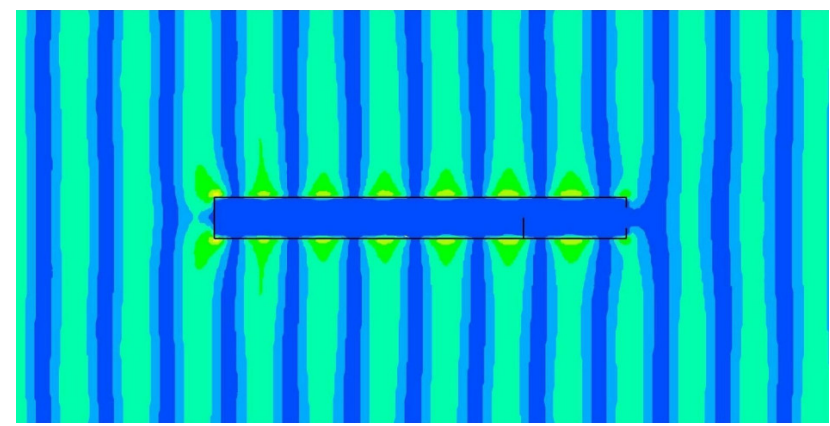

Fig. 3. Calculated distribution of electric field strength at $1 \mathrm{GHz}$.

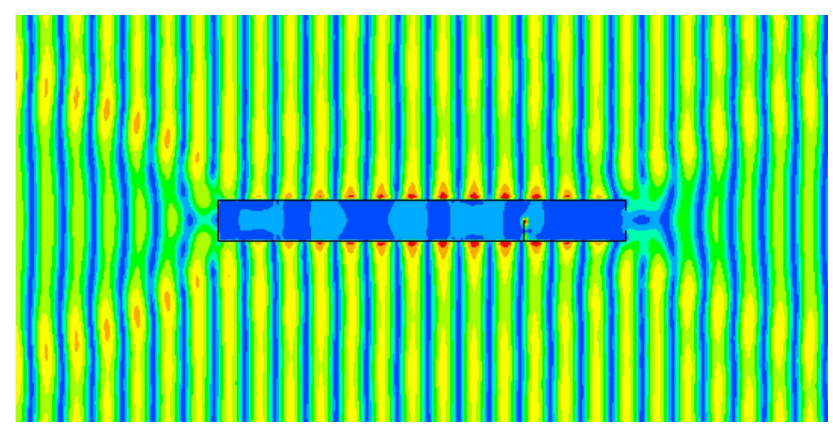

Fig. 4. Calculated distribution of electric field strength at $2 \mathrm{GHz}$.

$D_{\text {cyl }}=100 \mathrm{~mm}$, as mentioned in Sect. 2. The cylinder is equipped with an aperture (diameter $D_{a}=50 \mathrm{~mm}$ ) at the front side and a monopole probe positioned in a distance of $0.25 \mathrm{~m}$ with respect to the aperture. The monopole is implemented by an ideal conducting wire, which lengths is $l_{m}=50 \mathrm{~mm}$ and connected to the enclosure with a $50 \Omega$ resistor. The structure is illuminated by an incident electromagnetic plane wave as shown in Fig. 1. It should be mentioned that the monopole is orientated parallel to the vector of the electric field of the plane wave. The impedance voltage drop which is equivalent to the antenna base voltage of a field probe in measurements can calculated numerical by means of this simulation model as well as the field distribution inside and outside the missile enclosure.

\subsection{Simulation results}

All simulations have been performed in the frequency range between $10 \mathrm{MHz}$ and $4 \mathrm{GHz}$ at discrete frequencies. The step size was $10 \mathrm{MHz}$, thus 400 different frequencies have been treated within this investigation. The magnitude of the electric field strength of the incident plane wave is $E=1 \mathrm{kV} / \mathrm{m}$.

Figure 2 shows the calculated impedance voltage drop at the antenna base of the monopole. While the voltage drop is smaller than $1 \mathrm{~V}$ in the frequency range below $1.5 \mathrm{GHz}$, it increases rapidly at $1.8 \mathrm{GHz}$. In the frequency range above $1.8 \mathrm{GHz}$, the voltage drop is several times larger than $1 \mathrm{~V}$ for frequencies, but shows more or less strong variations. 


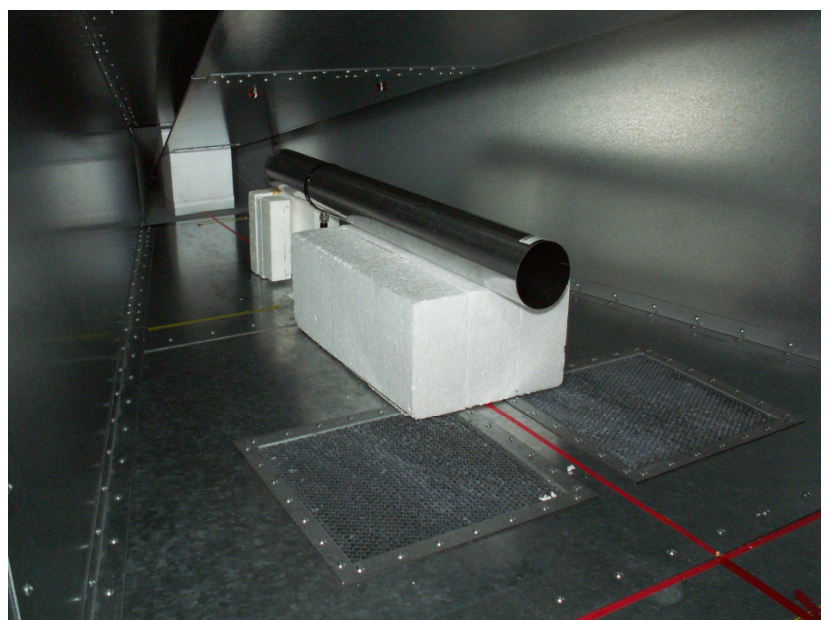

Fig. 5. Measurement setup inside the GTEM cell.

In order to find an explanation for this behaviour, the distribution of the electric field strength has been examined for two different frequencies. The calculated magnitude of field strength in the $\mathrm{x}-\mathrm{z}$-plane (with respect to the coordinate system in Fig. 1) is illustrated in Fig. 3 respectively in Fig. 4. While the magnitude is almost equal to the electric field in $\mathrm{z}$-direction, only the magnitude is presented. Although absolute field strength values are not given, both figures show the active principle very well.

As it could be seen in both figures, the incident wave propagates from the right side to the left one. While the wave propagation outside the hollow cylinder is nearly unaffected due to its presence at $1 \mathrm{GHz}$, field disturbances occur in front of the aperture at $2 \mathrm{GHz}$. Furthermore, the magnitude of the field strength inside the enclosure is negligible at the lower frequency, whereas wave propagation could be found inside the hollow cylinder at the higher frequency.

This frequency dependent behaviour is affected by the geometry of the simulation model, because the missile model forms a cylindrical waveguide. The cut-off frequency of a certain mode is the characteristic quantity of a waveguide. This quantity is given by Eq. (A1) in case of a transverse electrical mode ( $T E_{m n}$-mode) respectively by Eq. (A2) in case of a transverse magnetic mode $\left(\mathrm{TH}_{m n}\right.$-mode). Table 1 gives an overview about cut-off frequencies of the different modes which are able to propagate in a waveguide with $D=100 \mathrm{~mm}$. The fundamental mode is the $T E_{11}$-mode and the correspondent cut-off frequency is approximately $1.75 \mathrm{GHz}$.

Taking into consideration that the enclosure forms a cylindrical waveguide, the different field distributions can be explained easily. The incident electromagnetic wave couples to the waveguide because of aperture penetration at both investigated frequencies. While the cylindrical waveguides operates at $1 \mathrm{GHz}$ below the frequency cut-off, there is no wave propagation possible and the electromagnetic fields inside

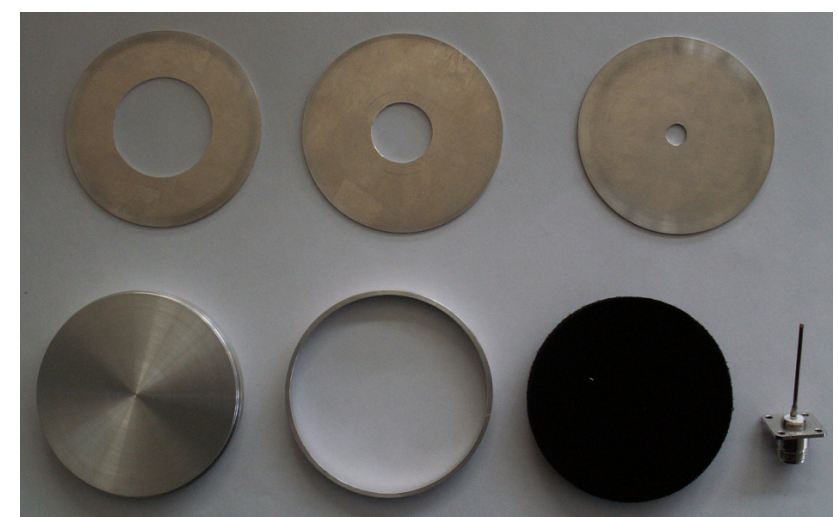

Fig. 6. Different apertures and terminations, monopole probe.

Table 1. Cut-off frequencies of a metallic tube with $D=100 \mathrm{~mm}$.

\begin{tabular}{lllll}
\hline Mode & $\begin{array}{l}\text { Bessel } \\
\text { function }\end{array}$ & $\begin{array}{l}\mathrm{n} \text {-th } \\
\text { root }\end{array}$ & $\begin{array}{l}\text { Function } \\
\text { Value }\end{array}$ & $\begin{array}{l}\text { Cut-off } \\
\text { frequency }\end{array}$ \\
\hline$T E_{11}$ & $J_{1}^{\prime}(x)$ & 1 & 1.841 & $1.757 \mathrm{GHz}$ \\
$T M_{01}$ & $J_{0}(x)$ & 1 & 2.405 & $2.295 \mathrm{GHz}$ \\
$T E_{21}$ & $J_{2}^{\prime}(x)$ & 1 & 3.054 & $2.914 \mathrm{GHz}$ \\
$T E_{11}$ & $J_{0}^{\prime}(x)$ & 1 & 3.832 & $3.657 \mathrm{GHz}$ \\
$T M_{11}$ & $J_{1}(x)$ & 1 & 3.832 & $3.657 \mathrm{GHz}$ \\
\hline
\end{tabular}

the metallic tube are dumped exponentially. The things are different in the higher frequency range due to the fact that the waveguide operates above its frequency cut-off. As illustrated in Fig. 4, the wave excited by aperture penetration propagates from the right side to the left one. The metallic cap on the left side forms a short termination of the waveguide, thus the wave is reflected completely. The reflected wave propagates in backward direction and reaches the aperture, where it is reflected partly, as well as the radiated. Thus, not only standing waves occur inside the enclosure, but also field disturbances are affected in front of the hollow cylinder.

\section{Measurements}

Measurements of the hollow cylinder have been preformed with continuous wave signals in the frequency domain. The aim of these measurements is the investigation of different aperture sizes and terminations.

\subsection{Measurement setup}

A GTEM cell is a well suited environment in order to generate defined electromagnetic fields. Thus, the measurements have been performed in a GTEM 1250 . Therefore, the metallic hollow cylinder is placed parallel to the ground plane of the cell as illustrated in Fig. 5. The aperture is oriented 


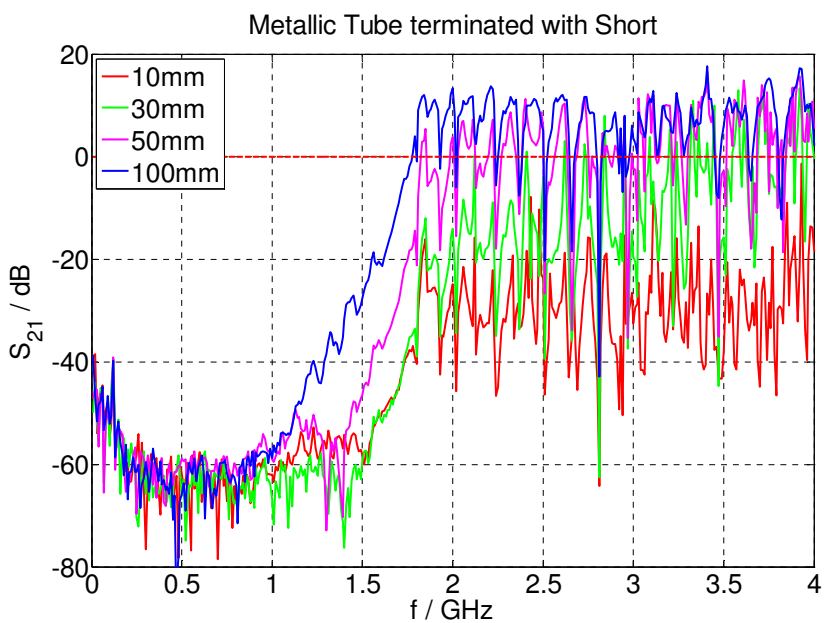

Fig. 7. Variation of the aperture size, tube terminated with short.

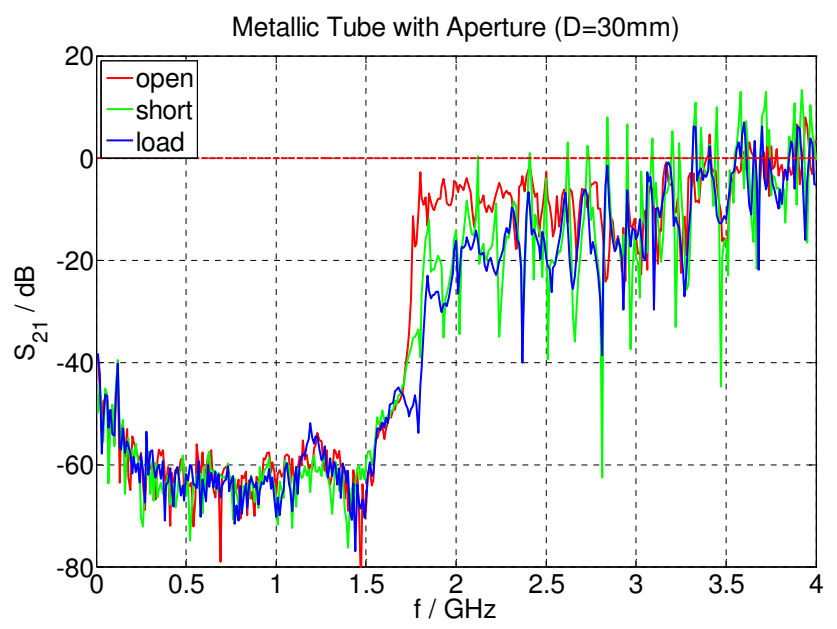

Fig. 8. Variation of the termination (aperture size $D_{a}=30 \mathrm{~mm}$ ).

towards the cell port and the open ending towards the absorbing cones. The monopole probe inside the metallic tube is positioned in vertical direction with respect to the ground plane, thus it measured the electric field in z-direction. Its length is $l_{m}=5 \mathrm{~mm}$ and it is positioned in a distance of $0.25 \mathrm{~m}$ to the aperture.

The metallic tube can be equipped with different apertures on one end and terminations on the other. Figure 6 shows the different apertures, which are realized due to metallic discs with centric holes. A metallic support fixes the different apertures at the front side of the tube. Possible terminations of the open ending of the tube are open, short and load. The load emulates the loading of the cylinder with a printed circuit board (PCB) and is implemented by absorbing foam with cylindrical shape. A network analyzer serves as power source as well as receiver. Therefore, one port of the network analyzer is connected to the cell port and the other to the monopole probe.

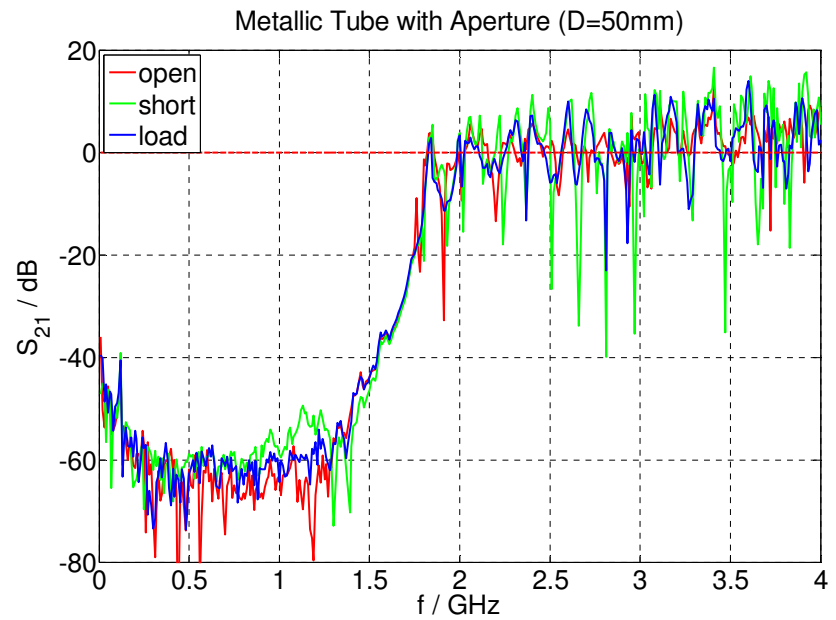

Fig. 9. Variation of the termination (aperture size $D_{a}=50 \mathrm{~mm}$ ).

\subsection{Measurement results}

All measurements have been performed with CW (continuous wave) signals in the frequency range from $10 \mathrm{MHz}$ up to $4 \mathrm{GHz}$ at 400 discrete frequencies. While the measured quantity is the transfer function between the cell port and the monopole, the frequency response of the monopole has to be taken into consideration. Thus, the $S_{21}$-parameter denotes the corrected transfer function in the following.

The influence of different terminations is shown in Fig. 8 for an aperture size of $D=30 \mathrm{~mm}$. The magnitude of the measured $S_{21}$-parameter is less than $-50 \mathrm{~dB}$ in the frequency range below $1.75 \mathrm{GHz}$ and nearly independent from the termination. At approximately $1.75 \mathrm{GHz}$, there is an abrupt rise of this parameter. While the termination with short shows alternating minimums and maximums in the higher frequency range, the deviation is smaller for a termination with open. Furthermore, the termination with load has got a lower magnitude than the termination open, because the absorbing foam dumps the resonance effects.

The rapid increase of the transfer function at approximately $1.75 \mathrm{GHz}$ is caused by the geometrical dimensions of the metallic tube, because the tube forms a cylindrical waveguide. The cut-off frequency of the fundamental mode is $1.757 \mathrm{GHz}$ with respect to Eq. (A1) and Table 1. Below $1.757 \mathrm{GHz}$, the electromagnetic field excited at the aperture is an evanescent field and dumped exponentially. An increase of the aperture size affects of a slow rise in the frequency range between $1.3 \mathrm{GHz}$ and $1.75 \mathrm{GHz}$, thus the abrupt increase at the cut-off frequency is more flat (see Fig. 7). Nevertheless, the magnitude is larger in the upper frequency range than in case of a smaller aperture, as shown in Fig. 9. Above the cut-off frequency of the fundamental mode, transverse electrical waves ( $T E_{11}$-mode) can be excited at the aperture. These waves propagate towards the termination, where they are reflected more or less depending on 
the termination. The reflected waves propagate towards the aperture, where the waves will be partially reflected respectively radiated to the surroundings. It is quite obvious that metallic tube operates as a cavity resonator, thus the magnitude of transfer function reaches values larger than $0 \mathrm{~dB}$ at certain frequencies. The termination with load effects a dumping of the resonances.

\section{Conclusions}

In this paper the shielding properties of a missile enclosure was examined by numerical field calculations as well as by measurements. Under assumption of a sufficient conductivity of the enclosure walls, the coupling to the inside is affected by aperture penetration. Therefore, the missile enclosure was emulated by means of a metallic hollow cylinder, which includes an aperture in the top cap. Furthermore, a monopole field probe was positioned inside the cylinder in order to detect the excited electric field inside.

The simulations point out the active principle very well. The metallic hollow cylinder behaves like a cylindrical waveguide. Below the first cut-off frequency, only evanescent fields are present in the waveguide. In the higher frequency range, the fundamental mode ( $T E_{11}$-mode) or other higher order modes are able to propagate. The calculated voltage at the antenna base of the monopole increases rapidly. While the enclosure is ideal conductive, the hollow cylinder forms a cavity with the high quality factor. The resonances will be dumped only by losses due to radiated emissions at the aperture.

The measurements show results, which are very similar to the numerical calculations ones. Below the first cut-off frequency, only evanescent fields are present in the waveguide. In the higher frequency range, the fundamental mode is able to propagate and the field strength increases rapidly. The variation of the aperture shows that a growing aperture size increases the field excitation in the higher frequency range above $1.75 \mathrm{GHz}$.

The different terminations of the waveguides influence the dumping of the standing waves. While a cylinder terminated with a metallic cap (short) shows the strongest resonance behaviour, an open ending effects a higher dumping due to additional radiated emissions. The most realistic setup, which includes a PCB model that consists of absorbing foam, shows the smallest resonance effects.

\section{Appendix A}

\section{Cut-off frequencies of cylindrical waveguides}

The characteristic quantity of a waveguide is the cut-off frequency $f_{c}$ of a certain mode. In case of a cylindrical waveguide it is given for transverse electrical modes $\left(T E_{m n}\right.$-modes) by

$f_{c, m n}^{T E}=\frac{c_{0}}{\pi \sqrt{\mu_{r} \varepsilon_{r}}} \cdot \frac{x_{m n}^{\prime}}{D}$,

where $m, n$ are integers and $D$ is the diameter of the metallic tube. $x_{m n}^{\prime}$ is the $n$-th root of the deviation of Bessel function $J_{m}^{\prime}(x) . \varepsilon_{r}$ and $\mu_{r}$ are the relative permittivity respectively permeability of the material inside the waveguide and $c_{0}$ is the speed of the light. In case of transverse magnetic modes ( $T M_{m n}$-modes), the cut-off frequency is dependent to the $n$ th root $x_{m n}$ of the of Bessel function $J_{m}(x)$ :

$f_{c, m n}^{T M}=\frac{c_{0}}{\pi \sqrt{\mu_{r} \varepsilon_{r}}} \cdot \frac{x_{m n}}{D}$.

Acknowledgements. This investigation is part of the study "Protection of Electronic Systems to electromagnetic Influences, Phase II", commissioned by the Armed Forces Scientific Institute for Protection Technologies and NBC Protection (Munster, Germany). The responsibility for the content is with the authors.

\section{References}

Collin, R. E.: Field theory of guided waves, IEEE Press, New York, 1991.

Groh, C., Kärst, J. P., Koch, M., and Garbe, H.: TEM Waveguides for EMC Measurements, IEEE Transactions on Electromagnetic Compatibility, 41(4), 440-445, Nov., 1999.

Nitsch, D., Camp, M., Sabath, F., ter Haseborg, J. L., and Garbe, H.: Susceptibility of Some Electronic Equipment to HPM Threats, IEEE Transactions on Electromagnetic Compatibility, 46(3), 380-389, Aug., 2004. 